\title{
Modeling and Simulation of the Vibration Characteristics of the In-Wheel Motor Driving Vehicle Based on Bond Graph
}

\author{
Di Tan and Qiang Wang \\ School of Transportation and Vehicle Engineering, Shandong University of Technology, Zibo 255049, China \\ Correspondence should be addressed to Di Tan; tandi@sdut.edu.cn
}

Received 12 July 2015; Revised 18 September 2015; Accepted 11 October 2015

Academic Editor: Matteo Aureli

Copyright (C) 2016 D. Tan and Q. Wang. This is an open access article distributed under the Creative Commons Attribution License, which permits unrestricted use, distribution, and reproduction in any medium, provided the original work is properly cited.

\begin{abstract}
Bond graph theory is applied to the modeling and analysis of the vibration characteristics of the in-wheel motor driving vehicle. First, an 11-degree-of-freedom vibration model of the in-wheel motor driving vehicle is established based on bond graph, and then the correctness of the model is verified. Second, under the driving condition of class B road excitations and a speed of $50 \mathrm{Km} / \mathrm{h}$, the vibration characteristics of the in-wheel motor driving vehicle are simulated and analyzed, and the activity of each part in the system is then calculated. Third, these parts that have less of an effect on the vibration characteristics of an in-wheel motor driving vehicle are identified according to the magnitude of the activity, and then the model is simplified by removing these parts. Finally, the reliability of the simplified model is verified by comparing the vibration characteristics of the model before and after simplification. This study can provide a method for the modeling and simulation of the vibration characteristics of the in-wheel motor driving vehicle.
\end{abstract}

\section{Introduction}

Vehicle vibration has always been a popular research topic in the field of vehicles, because the analysis of the vehicle vibration characteristics can effectively evaluate the vehicle ride performance. At present, most studies adopt Newton's second law method to model and analyze the vibration problems of vehicles.

The bond graph (BG) method was proposed by American H. M. Paynter, a professor in the late 1950s. The BG method can be used to solve the modeling problem of the system involved in the multienergy field from the power perspective [1]. Compared with the traditional modeling method, BG theory has the following advantages: (1) the BG theory can be applied in a unified way to address the multienergy forms of the system; (2) the modular modeling method simplifies the modeling of the complex system; and (3) based on the modeling idea on the energy transfer, it is easy to simplify the model [2]. Currently, only a few researchers have performed research using the BG method in the field of vehicle vibration. In one study [3], a 5-degree-of-freedom vibration model for a traditional vehicle was established based on the BG method, and the simulation results were compared with real vehicle test data. In addition, the BG method was used to establish the power train model of an electric vehicle with a traditional chassis structure and deduce the state equation of system [4]. The BG theory was also applied for the analysis of the ride comfort of the terrain crane, and a 2-degreeof-freedom vibration model was established using the BG method [5]. In another study [6], the active hydropneumatic suspension system of a multiaxle vehicle with a traditional chassis was analyzed based on the BG method. In summary, BG theory has been applied to vehicle dynamics analysis to some extent, but most of the research has focused on traditional vehicles. For the in-wheel motor driving vehicle (IWMDV), the chassis structure is different compared to that of the traditional vehicles; in addition, the motor, speed reducer, and brakes are all integrated into the wheel. The BG method is a physical object-oriented method. The difference in the chassis structure represents the differences in the physical and the BG model. 


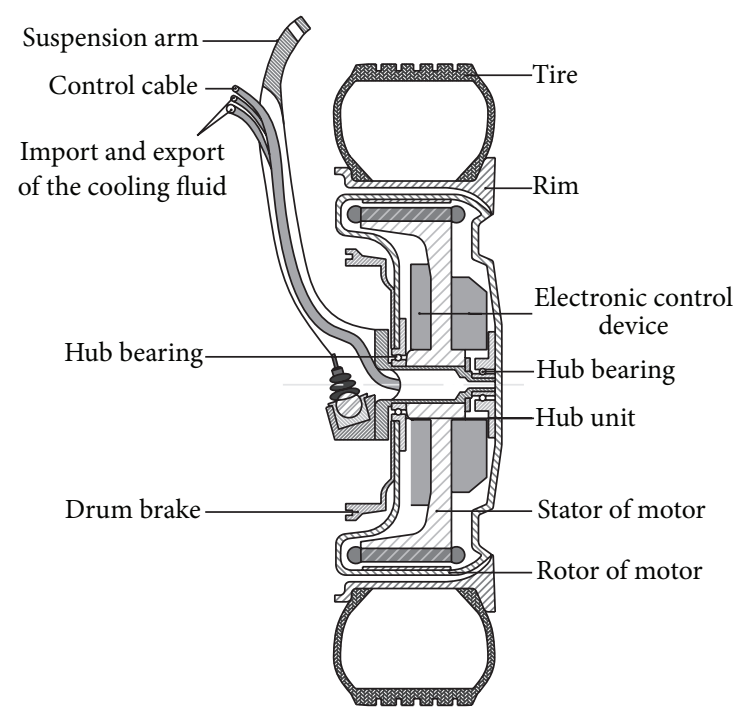

FIgURE 1: Basic structure of the electric wheel.

Based on the above advantages, the BG theory is applied to the vibration analysis of the IWMDV in this paper. An 11-degree-of-freedom IWMDV physical model is developed first using the BG method for modular modeling and assembly. Next, the mathematical model is deduced, for which the validity is verified using Newton's second law. Based on the developed model, the vibration characteristics of the IWMDV are analyzed, and the activity of each part is calculated in the system. Taking advantage of the BG method, model reduction and model simplification are performed, and several of the parts that have little influence on the vehicle vibration are removed from the original physical model. Furthermore, the reliability of the model after simplification (MAS) is verified by the comparison analysis with the model before simplification (MBS).

\section{Vehicle Vibration Model}

2.1. Electric Wheel Structure. As shown in Figure 1, the basic structure of the electric wheel is mainly composed of the following components: a motor stator, a motor rotor, brakes, wheel hub bearings, a rim, and a tire. In this structure, the motor housing and the rim are directly combined in one body; therefore, by controlling the motor rotor rotation, the vehicle can be directly driven [7].

2.2. Physical Model of the Vehicle. To facilitate analysis, the following hypotheses are applied to the vehicle physical model:

(1) the vehicle body is regarded as a rigid body;

(2) the operating conditions are set for uniform linear driving;

(3) the wheel hub bearing is equivalent to a springdamper;

(4) the spring force is simplified as a linear function of displacement, and the damping force is simplified as a linear function of velocity;
(5) the vehicle body has 3 degrees of freedom (vertical, pitch, and roll), and the unsprung mass has 8 degrees of freedom in the vertical direction; that is, the vehicle has a total of 11 degrees of freedom.

Using the above hypotheses, the 11-degree-of-freedom physical model is established, as shown in Figure 2.

In Figure 2, $m_{b}$ is the body mass; $l_{f}$ is the distance from the center of the body mass to the front axle; $l_{r}$ is the distance from the center of the body mass to the rear axle; $d$ is the track width; $\theta$ is the vehicle pitching angle; $\varphi$ is the vehicle roll angle; $k_{i 1}$ and $k_{i 4}$ are the tire stiffness and the suspension stiffness, respectively; $c_{i 1}$ and $c_{i 4}$ are the tire damping and the suspension damping, respectively; $m_{i 1}$ is the total mass of the tire, rim, and motor rotor; $m_{i 2}$ is the total mass of the motor stator and the brake, and so forth; $k_{i 2}$ and $k_{i 3}$ are the bearing stiffness; $c_{i 2}$ and $c_{i 3}$ are the bearing damping; $z_{i 1}$ and $z_{i 2}$ are the vertical displacements of the corresponding mass; $z_{i}$ is the vertical displacement of the connection point between the suspension and vehicle body; $i=a, b, c$, and $d$ represents the right front wheel, left front wheel, right rear wheel, and left rear wheel, respectively; and $z_{j}$ is the displacement input of the road surface roughness, where $j=1,2,3$, and 4 .

\subsection{The BG Model of the Vehicle}

2.3.1. Basic BG Modeling Method. For a mechanical system, the BG model includes five basic elements: $\mathrm{R}$ element represents damping, $\mathrm{C}$ element represents spring, I element represents mass or inertia, Se element represents the force source, and Sf element represents the velocity source; $\mathrm{R}$ element is the energy dissipation element and $\mathrm{C}$ and I elements are the energy storage elements. The five elements are linked by constant velocity 1 -junctions, constant force 0 -junctions, and transformer TF-junctions. The basic BG modeling method is as follows:

(1) Create 1-junctions for each absolute velocity and relative velocity in the model.

(2) Create 0 -junctions and transformer TF-junctions between correlative 1-junctions to establish the relationships between the correlative absolute velocities and between the correlative relative velocity and absolute velocity using the relationship between the velocities with power flow direction.

(3) Connect the five basic elements to the corresponding 1 -junctions with suitable causality.

2.3.2. Modular Modeling. Modular modeling not only makes the establishment of the model clearer and easier but also makes modification of the model more convenient. The vehicle physical model shown in Figure 2 is modeled using the BG method.

In this modeling approach, a model can be divided into several subsystems; each subsystem is modeled, and then all subsystem models are combined to obtain the whole BG model of the vehicle [8]. The physical model shown in Figure 2 can be divided into five subsystems: the body system and four quarter car suspension systems. First, the BG models 


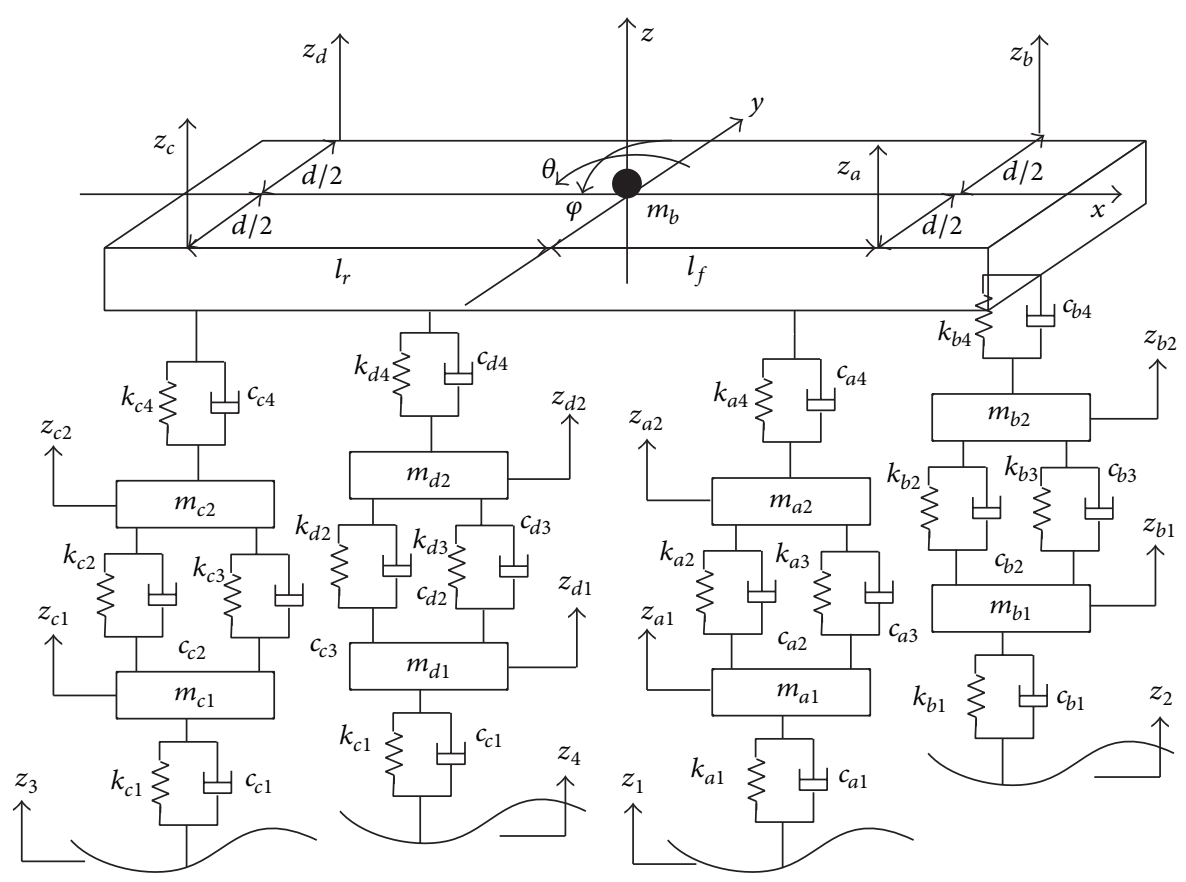

FIGURE 2: 11-degree-of-freedom physical model.

of the left front (LF), right front (RF), left rear (LR), and right rear (RR) quarter car suspension systems are set up, as shown in Figure 3.

Next, the body system BG model is established, as shown in Figure 4.

2.3.3. BG Model Combination. The reserved ports LF, RF, LR, and RR in Figure 3 are first connected with the corresponding ports LF, RF, LR, and RR in Figure 4. Then, the whole BG model of the vehicle can be obtained and further simplified as shown in Figure 5.

\subsection{Mathematical Model of the Vehicle}

2.4.1. Definition of the BG Symbols. Using the generalized variables defined in BG theory [1], for mechanical translation, the generalized variables $q$ and $p$ represent displacement and momentum, respectively, whereas, for mechanical rotation, $q$ and $p$ represent angle and angular momentum, respectively. In addition, the generalized variables $\dot{q}$ and $\dot{p}$ are the derivatives of $q$ and $p$ with respect to time, respectively. Likewise, for machine translation, $\dot{q}$ and $\dot{p}$ represent velocity and force, respectively, and for mechanical rotation, $\dot{q}$ and $\dot{p}$ represent angular velocity and torque, respectively.

Each element in the BG model has a subscript, and each subscript corresponds to a different element. The elements $q$, $p, \dot{q}$, and $\dot{p}$ with different subscripts refer to the corresponding symbol meaning of the corresponding element.

2.4.2. State Equation of the BG Model. According to the BG model of the vehicle, the causality and power flow direction are analyzed by the BG method, and then the vehicle state equation of the BG model is expressed as follows [9].
The vertical velocity equation of $m_{a 1}$ is

$$
\dot{q}_{4}=\dot{q}_{a}-\frac{p_{6}}{m_{a 1}} .
$$

The vertical velocity equation of $m_{a 2}$ is

$$
\dot{q}_{14}=\dot{q}_{12}=\frac{p_{6}}{m_{a 1}}-\frac{p_{17}}{m_{a 2}} .
$$

The vertical velocity equation of $m_{b 1}$ is

$$
\dot{q}_{65}=\dot{q}_{b}-\frac{p_{60}}{m_{b 1}} .
$$

The vertical velocity equation of $m_{b 2}$ is

$$
\dot{q}_{56}=\dot{q}_{53}=\frac{p_{60}}{m_{b 1}}-\frac{p_{49}}{m_{b 2}} .
$$

The vertical velocity equation of $m_{c 1}$ is

$$
\dot{q}_{106}=\dot{q}_{c}-\frac{p_{102}}{m_{c 1}} .
$$

The vertical velocity equation of $m_{c 2}$ is

$$
\dot{q}_{99}=\dot{q}_{96}=\frac{p_{102}}{m_{c 1}}-\frac{p_{91}}{m_{c 2}} .
$$

The vertical velocity equation of $m_{d 1}$ is

$$
\dot{q}_{85}=\dot{q}_{d}-\frac{p_{81}}{m_{d 1}} .
$$

The vertical velocity equation of $m_{d 2}$ is

$$
\dot{q}_{75}=\dot{q}_{77}=\frac{p_{81}}{m_{d 1}}-\frac{p_{70}}{m_{d 2}} .
$$




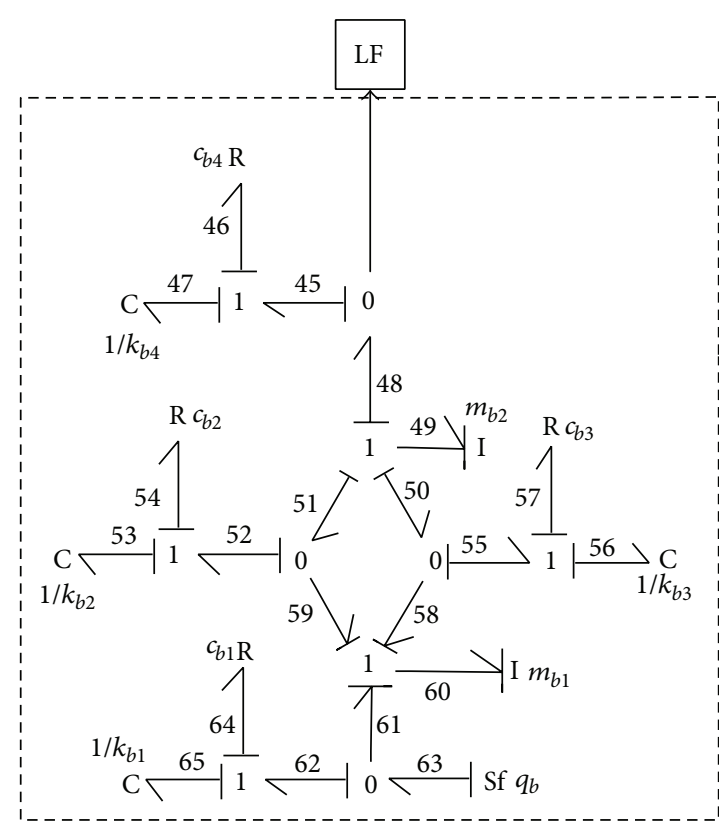

(a) LF

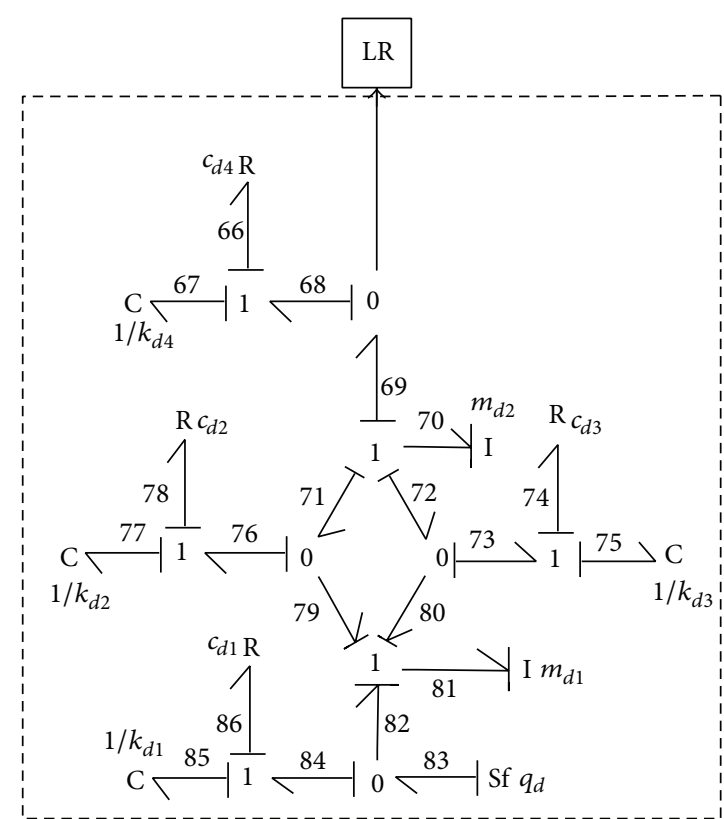

(c) LR

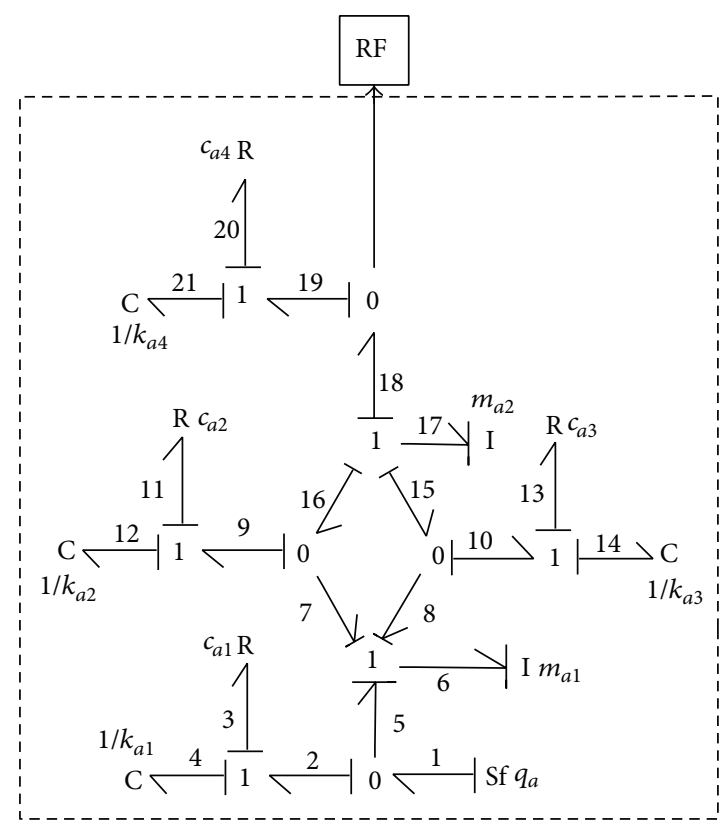

(b) RF

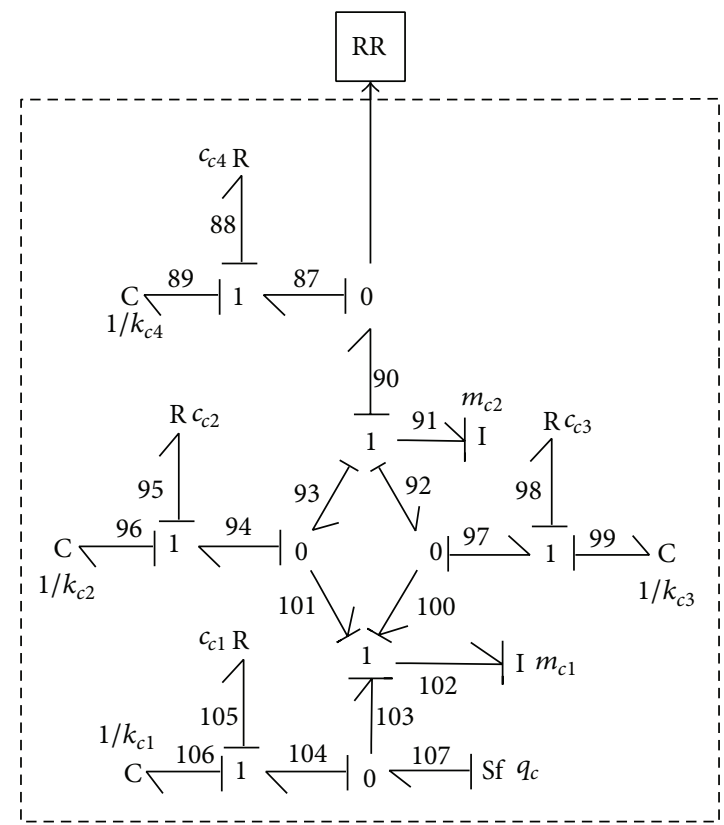

(d) $\mathrm{RR}$

FIGURE 3: BG model of the four quarter car suspension systems.

The vertical motion equation of the four endpoints of the vehicle body can be obtained:

$$
\begin{aligned}
& \dot{q}_{21}=\frac{p_{17}}{m_{a 2}}-l_{f} \frac{p_{26}}{I_{y}}-\frac{d}{2} \frac{p_{44}}{I_{x}}-\frac{p_{35}}{m_{b}}, \\
& \dot{q}_{47}=\frac{p_{49}}{m_{b 2}}-l_{f} \frac{p_{26}}{I_{y}}+\frac{d}{2} \frac{p_{44}}{I_{x}}-\frac{p_{35}}{m_{b}}, \\
& \dot{q}_{89}=\frac{p_{91}}{m_{c 2}}+l_{r} \frac{p_{26}}{I_{y}}-\frac{d}{2} \frac{p_{44}}{I_{x}}-\frac{p_{35}}{m_{b}},
\end{aligned}
$$

$$
\dot{q}_{67}=\frac{p_{70}}{m_{d 2}}+l_{r} \frac{p_{26}}{I_{y}}+\frac{d}{2} \frac{p_{44}}{I_{x}}-\frac{p_{35}}{m_{b}} .
$$

The vertical force equation of $m_{a 1}$ is

$$
\dot{p}_{6}=c_{a 1} \dot{q}_{4}+k_{a 1} q_{4}-c_{a 2} \dot{q}_{12}-k_{a 2} q_{12}-c_{a 3} \dot{q}_{14}-k_{a 3} q_{14} .
$$

The vertical force equation of $m_{a 2}$ is

$$
\begin{aligned}
\dot{p}_{17}= & c_{a 2} \dot{q}_{12}+k_{a 2} q_{12}+c_{a 3} \dot{q}_{14}+k_{a 3} q_{14}-c_{a 4} \dot{q}_{21} \\
& -k_{a 4} q_{21} .
\end{aligned}
$$




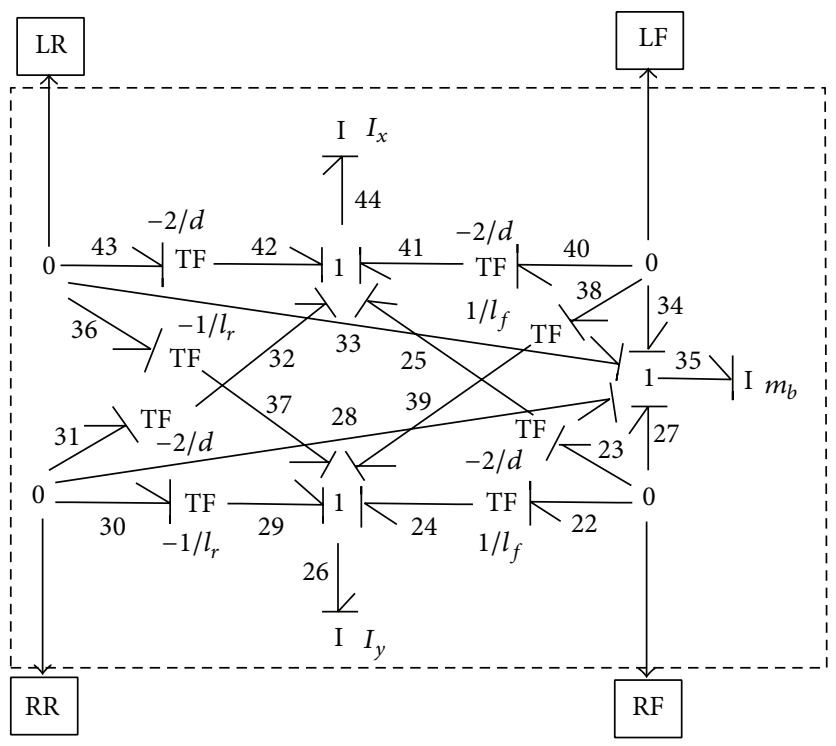

FIGURE 4: BG model of the vehicle body system.

The vertical force equation of $m_{b 1}$ is

$$
\begin{aligned}
\dot{p}_{60}= & c_{b 1} \dot{q}_{65}+k_{b 2} q_{65}-c_{b 3} \dot{q}_{56}-k_{b 3} q_{56}-c_{b 2} \dot{q}_{53} \\
& -k_{b 2} q_{53} .
\end{aligned}
$$

The vertical force equation of $m_{b 2}$ is

$$
\begin{aligned}
\dot{p}_{49}= & c_{b 3} \dot{q}_{56}+k_{b 3} q_{56}+c_{b 2} \dot{q}_{53}+k_{b 2} q_{53}-c_{b 4} \dot{q}_{47} \\
& -k_{b 4} q_{47} .
\end{aligned}
$$

The vertical force equation of $m_{c 1}$ is

$$
\begin{aligned}
\dot{p}_{102}= & c_{c 1} \dot{q}_{106}+k_{c 1} q_{106}-c_{c 2} \dot{q}_{96}-k_{c 2} q_{96}-c_{c 3} \dot{q}_{99} \\
& -k_{c 3} q_{99} .
\end{aligned}
$$

The vertical force equation of $m_{c 2}$ is

$$
\begin{aligned}
\dot{p}_{91}= & c_{c 2} \dot{q}_{96}+k_{c 2} q_{96}+c_{c 3} \dot{q}_{99}+k_{c 3} q_{99}-c_{c 4} \dot{q}_{89} \\
& -k_{c 4} q_{89} .
\end{aligned}
$$

The vertical force equation of $m_{d 1}$ is

$$
\begin{aligned}
\dot{p}_{81}= & c_{d 1} \dot{q}_{85}+k_{d 1} q_{85}-c_{d 2} \dot{q}_{77}-k_{d 2} q_{77}-c_{d 3} \dot{q}_{75} \\
& -k_{d 3} q_{75} .
\end{aligned}
$$

The vertical force equation of $m_{d 2}$ is

$$
\begin{aligned}
\dot{p}_{70}= & c_{d 2} \dot{q}_{77}+k_{d 2} q_{77}+c_{d 3} \dot{q}_{75}+k_{d 3} q_{75}-c_{d 4} \dot{q}_{67} \\
& -k_{d 4} q_{67} .
\end{aligned}
$$
follows:

The vertical force equation of the vehicle body is as

$$
\begin{aligned}
\dot{p}_{35}= & c_{a 4} \dot{q}_{21}+k_{a 4} q_{21}+c_{c 4} \dot{q}_{89}+k_{c 4} q_{89}+c_{d 4} \dot{q}_{67} \\
& +k_{d 4} q_{67}+c_{b 4} \dot{q}_{47}+k_{b 4} q_{47} .
\end{aligned}
$$

The pitching moment equation of the vehicle body is

$$
\begin{aligned}
\dot{p}_{26}= & l_{f}\left(c_{a 4} \dot{q}_{21}+k_{a 4} q_{21}+c_{b 4} \dot{q}_{47}+k_{b 4} q_{47}\right) \\
& -l_{r}\left(c_{c 4} \dot{q}_{89}+k_{c 4} q_{89}+c_{d 4} \dot{q}_{67}+k_{d 4} q_{67}\right) .
\end{aligned}
$$

The roll torque equation of the vehicle body is

$$
\begin{aligned}
\dot{p}_{44} & =\frac{d}{2}\left(c_{a 4} \dot{q}_{21}+k_{a 4} q_{21}+c_{c 4} \dot{q}_{89}+k_{c 4} q_{89}-c_{d 4} \dot{q}_{67}\right. \\
& \left.-k_{d 4} q_{67}-c_{b 4} \dot{q}_{47}-k_{b 4} q_{47}\right) .
\end{aligned}
$$

\section{Validation of the BG Model}

3.1. The Mathematical Model Developed Using the Newton Method. Based on the physical model in Figure 2, the mathematical model is derived using Newton's second law; a description is given as follows.

The vertical motion equations of the unsprung masses are

$$
\begin{aligned}
m_{a 1} \ddot{z}_{a 1}= & c_{a 1}\left(\dot{z}_{1}-\dot{z}_{a 1}\right)+k_{a 1}\left(z_{1}-z_{a 1}\right) \\
& -\left(c_{a 2}+c_{a 3}\right)\left(\dot{z}_{a 1}-\dot{z}_{a 2}\right) \\
& -\left(k_{a 2}+k_{a 3}\right)\left(z_{a 1}-z_{a 2}\right), \\
m_{a 2} \ddot{z}_{a 2}= & \left(c_{a 2}+c_{a 3}\right)\left(\dot{z}_{a 1}-\dot{z}_{a 2}\right) \\
& +\left(k_{a 2}+k_{a 3}\right)\left(z_{a 1}-z_{a 2}\right) \\
& -c_{a 4}\left(\dot{z}_{a 2}-\dot{z}_{a}\right)-k_{a 4}\left(z_{a 2}-z_{a}\right),
\end{aligned}
$$




$$
\begin{aligned}
& m_{b 1} \ddot{z}_{b 1}=c_{b 1}\left(\dot{z}_{2}-\dot{z}_{b 1}\right)+k_{b 1}\left(z_{2}-z_{b 1}\right) \\
& -\left(c_{b 3}+c_{b 2}\right)\left(\dot{z}_{b 1}-\dot{z}_{b 2}\right) \\
& -\left(k_{b 3}+k_{b 2}\right)\left(z_{b 1}-z_{b 2}\right), \\
& m_{b 2} \ddot{z}_{b 2}=\left(c_{b 2}+c_{b 3}\right)\left(\dot{z}_{b 1}-\dot{z}_{b 2}\right) \\
& +\left(k_{b 2}+k_{b 3}\right)\left(z_{b 1}-z_{b 2}\right)-c_{b 4}\left(\dot{z}_{b 2}-\dot{z}_{b}\right) \\
& -k_{b 4}\left(z_{b 2}-z_{b}\right) \\
& m_{c 1} \ddot{z}_{c 1}=c_{c 1}\left(\dot{z}_{3}-\dot{z}_{c 1}\right)+k_{c 1}\left(z_{3}-z_{c 1}\right) \\
& -\left(c_{c 3}+c_{c 2}\right)\left(\dot{z}_{c 1}-\dot{z}_{c 2}\right) \\
& -\left(k_{c 3}+k_{c 2}\right)\left(z_{c 1}-z_{c 2}\right), \\
& m_{c 2} \ddot{z}_{c 2}=\left(c_{c 2}+c_{c} 3\right)\left(\dot{z}_{c 1}-\dot{z}_{c 2}\right) \\
& +\left(k_{c 2}+k_{c 3}\right)\left(z_{c 1}-z_{c 2}\right)-c_{c 4}\left(\dot{z}_{c 2}-\dot{z}_{c}\right) \\
& -k_{c 4}\left(z_{c 2}-z_{c}\right) \\
& m_{d 1} \ddot{z}_{d 1}=c_{d 1}\left(\dot{z}_{4}-\dot{z}_{d 1}\right)+k_{d 1}\left(z_{4}-z_{d 1}\right) \\
& -\left(c_{d 3}+c_{d 2}\right)\left(\dot{z}_{d 1}-\dot{z}_{d 2}\right) \\
& -\left(k_{d 3}+k_{d 2}\right)\left(z_{d 1}-z_{d 2}\right), \\
& m_{d 2} \ddot{z}_{d 2}=\left(c_{d 2}+c_{d 3}\right)\left(\dot{z}_{d 1}-\dot{z}_{d 2}\right) \\
& +\left(k_{d 2}+k_{d 3}\right)\left(z_{d 1}-z_{d 2}\right) \\
& -c_{d 4}\left(\dot{z}_{d 2}-\dot{z}_{d}\right)-k_{d 4}\left(z_{d 2}-z_{d}\right) .
\end{aligned}
$$

The vertical, pitch, and roll motion equations of the vehicle body are

$$
\begin{aligned}
& m_{b} \ddot{z}=c_{a 4}\left(\dot{z}_{a 2}-\dot{z}_{a}\right)+k_{a 4}\left(z_{a 2}-z_{a}\right)+c_{b 4}\left(\dot{z}_{b 2}-\dot{z}_{b}\right) \\
& \quad+k_{b 4}\left(z_{b 2}-z_{b}\right)+c_{c 4}\left(\dot{z}_{c 2}-\dot{z}_{c}\right)+k_{c 4}\left(z_{c 2}-z_{c}\right) \\
& \quad+c_{d 4}\left(\dot{z}_{d 2}-\dot{z}_{d}\right)+k_{d 4}\left(z_{d 2}-z_{d}\right) \\
& I_{y} \ddot{\theta}=l_{f}\left[c_{a 4}\left(\dot{z}_{a 2}-\dot{z}_{a}\right)+k_{a 4}\left(z_{a 2}-z_{a}\right)\right. \\
& \left.\quad+c_{b 4}\left(\dot{z}_{b 2}-\dot{z}_{b}\right)-k_{b 4}\left(z_{b 2}-z_{b}\right)\right]-l_{r}\left[c_{c 4}\left(\dot{z}_{c 2}-\dot{z}_{c}\right)\right. \\
& \left.\quad+k_{c 4}\left(z_{c 2}-z_{c}\right)+c_{d 4}\left(\dot{z}_{d 2}-\dot{z}_{d}\right)-k_{d 4}\left(z_{d 2}-z_{d}\right)\right] \\
& I_{x} \ddot{\phi}=\frac{d}{2}\left[c_{a 4}\left(\dot{z}_{a 2}-\dot{z}_{a}\right)+k_{a 4}\left(z_{a 2}-z_{a}\right)\right. \\
& \quad+c_{c 4}\left(\dot{z}_{c 2}-\dot{z}_{c}\right)+k_{c 4}\left(z_{c 2}-z_{c}\right)-c_{b 4}\left(\dot{z}_{b 2}-\dot{z}_{b}\right) \\
& \left.\quad-k_{b 4}\left(z_{b 2}-z_{b}\right)-c_{d 4}\left(\dot{z}_{d 2}-\dot{z}_{d}\right)-k_{d 4}\left(z_{d 2}-z_{d}\right)\right] .
\end{aligned}
$$

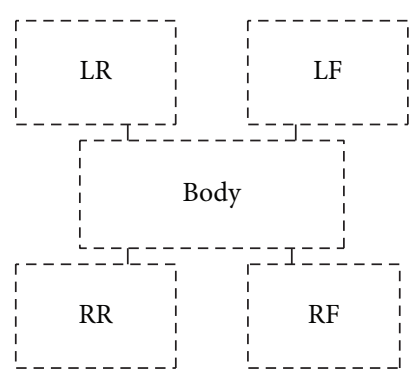

Figure 5: The whole BG model of the vehicle.

The vertical motion equations of the four endpoints of the vehicle body are

$$
\begin{aligned}
& \dot{z}_{a}=\dot{z}+l_{f} \dot{\theta}+\frac{1}{2} d \dot{\varphi}, \\
& \dot{z}_{b}=\dot{z}+l_{f} \dot{\theta}-\frac{1}{2} d \dot{\varphi}, \\
& \dot{z}_{c}=\dot{z}-l_{r} \dot{\theta}+\frac{1}{2} d \dot{\varphi}, \\
& \dot{z}_{d}=\dot{z}-l_{r} \dot{\theta}-\frac{1}{2} d \dot{\varphi} .
\end{aligned}
$$

3.2. Model Validation. To verify the correctness of the BG model in Figure 5, the state equations deduced by the BG model are translated into the form of motion equations deduced by Newton's second law. The transformation method is based on the relationship between the symbol in the state equations and the symbol in the Newtonian equations; accordingly, the state equations are converted into the Newtonian equations.

From (1), (2), and (13), the following expression is obtained:

$$
\begin{aligned}
m_{a 1} \ddot{z}_{a 1}= & c_{a 1}\left(\dot{z}_{1}-\dot{z}_{a 1}\right)+k_{a 1}\left(z_{1}-z_{a 1}\right) \\
& -\left(c_{a 2}+c_{a 3}\right)\left(\dot{z}_{a 1}-\dot{z}_{a 2}\right) \\
& -\left(k_{a 2}+k_{a 3}\right)\left(z_{a 1}-z_{a 2}\right) .
\end{aligned}
$$

From (2), (9), and (14), the following expression is obtained:

$$
\begin{aligned}
m_{a 2} \ddot{z}_{a 2}= & -c_{a 4}\left(\dot{z}_{a 2}-\dot{z}_{a}\right)-k_{a 4}\left(z_{a 2}-z_{a}\right) \\
& +\left(c_{a 2}+c_{a 3}\right)\left(\dot{z}_{a 1}-\dot{z}_{a 2}\right) \\
& +\left(k_{a 2}+k_{a 3}\right)\left(z_{a 1}-z_{a 2}\right) .
\end{aligned}
$$

From (3), (4), and (15), the following expression is obtained:

$$
\begin{aligned}
m_{b 1} \ddot{z}_{b 1}= & c_{b 1}\left(\dot{z}_{2}-\dot{z}_{b 1}\right)+k_{b 1}\left(z_{2}-z_{b 1}\right) \\
& -\left(c_{b 3}+c_{b 2}\right)\left(\dot{z}_{b 1}-\dot{z}_{b 2}\right) \\
& -\left(k_{b 3}+k_{b 2}\right)\left(z_{b 1}-z_{b 2}\right)
\end{aligned}
$$


From (4), (10), and (16), the following expression is obtained:

$$
\begin{aligned}
m_{b 2} \ddot{z}_{b 2}= & -c_{b 4}\left(\dot{z}_{b 2}-\dot{z}_{b}\right)-k_{b 4}\left(z_{b 2}-z_{b}\right) \\
& +\left(c_{b 2}+c_{b 3}\right)\left(\dot{z}_{b 1}-\dot{z}_{b 2}\right) \\
& +\left(k_{b 2}+k_{b 3}\right)\left(z_{b 1}-z_{b 2}\right) .
\end{aligned}
$$

From (5), (6), and (17), the following expression is obtained:

$$
\begin{aligned}
m_{c 1} \ddot{z}_{c 1}= & c_{c 1}\left(\dot{z}_{3}-\dot{z}_{c 1}\right)+k_{c 1}\left(z_{3}-z_{c 1}\right) \\
& -\left(c_{c 3}+c_{c 2}\right)\left(\dot{z}_{c 1}-\dot{z}_{c 2}\right) \\
& -\left(k_{c 3}+k_{c 2}\right)\left(z_{c 1}-z_{c 2}\right) .
\end{aligned}
$$

From (6), (11), and (18), the following expression is obtained:

$$
\begin{aligned}
m_{c 2} \ddot{z}_{c 2}= & -c_{c 4}\left(\dot{z}_{c 2}-\dot{z}_{c}\right)-k_{c 4}\left(z_{c 2}-z_{c}\right) \\
& +\left(c_{c 2}+c_{c} 3\right)\left(\dot{z}_{c 1}-\dot{z}_{c 2}\right) \\
& +\left(k_{c 2}+k_{c 3}\right)\left(z_{c 1}-z_{c 2}\right) .
\end{aligned}
$$

From (7), (8), and (19), the following expression is obtained:

$$
\begin{aligned}
m_{d 1} \ddot{z}_{d 1}= & c_{d 1}\left(\dot{z}_{4}-\dot{z}_{d 1}\right)+k_{d 1}\left(z_{4}-z_{d 1}\right) \\
& -\left(c_{d 3}+c_{d 2}\right)\left(\dot{z}_{d 1}-\dot{z}_{d 2}\right) \\
& -\left(k_{d 3}+k_{d 2}\right)\left(z_{d 1}-z_{d 2}\right) .
\end{aligned}
$$

From (8), (12), and (20), the following expression is obtained:

$$
\begin{aligned}
m_{d 2} \ddot{z}_{d 2}= & -c_{d 4}\left(\dot{z}_{d 2}-\dot{z}_{d}\right)-k_{d 4}\left(z_{d 2}-z_{d}\right) \\
& +\left(c_{d 2}+c_{d 3}\right)\left(\dot{z}_{d 1}-\dot{z}_{d 2}\right) \\
& +\left(k_{d 2}+k_{d 3}\right)\left(z_{d 1}-z_{d 2}\right) .
\end{aligned}
$$

From (9), (10), (11), (12), and (21), the vertical motion equation of the vehicle body is obtained:

$$
\begin{aligned}
m_{b} \ddot{z}= & c_{a 4}\left(\dot{z}_{a 2}-\dot{z}_{a}\right)+k_{a 4}\left(z_{a 2}-z_{a}\right)+c_{b 4}\left(\dot{z}_{b 2}-\dot{z}_{b}\right) \\
& +k_{b 4}\left(z_{b 2}-z_{b}\right)+c_{c 4}\left(\dot{z}_{c 2}-\dot{z}_{c}\right) \\
& +k_{c 4}\left(z_{c 2}-z_{c}\right)+c_{d 4}\left(\dot{z}_{d 2}-\dot{z}_{d}\right) \\
& +k_{d 4}\left(z_{d 2}-z_{d}\right) .
\end{aligned}
$$

From (9), (10), (11), (12), and (22), the pitch motion equation of the vehicle body is obtained:

$$
\begin{aligned}
I_{y} \ddot{\theta} & =l_{f}\left[c_{a 4}\left(\dot{z}_{a 2}-\dot{z}_{a}\right)+k_{a 4}\left(z_{a 2}-z_{a}\right)\right. \\
& \left.+c_{b 4}\left(\dot{z}_{b 2}-\dot{z}_{b}\right)-k_{b 4}\left(z_{b 2}-z_{b}\right)\right]-l_{r}\left[c_{c 4}\left(\dot{z}_{c 2}-\dot{z}_{c}\right)\right. \\
& \left.+k_{c 4}\left(z_{c 2}-z_{c}\right)+c_{d 4}\left(\dot{z}_{d 2}-\dot{z}_{d}\right)-k_{d 4}\left(z_{d 2}-z_{d}\right)\right] .
\end{aligned}
$$

From (9), (10), (11), (12), and (23), the roll motion equation of the vehicle body is obtained:

$$
\begin{aligned}
I_{x} \ddot{\phi} & =\frac{d}{2}\left[c_{a 4}\left(\dot{z}_{a 2}-\dot{z}_{a}\right)+k_{a 4}\left(z_{a 2}-z_{a}\right)\right. \\
& +c_{c 4}\left(\dot{z}_{c 2}-\dot{z}_{c}\right)+k_{c 4}\left(z_{c 2}-z_{c}\right)-c_{b 4}\left(\dot{z}_{b 2}-\dot{z}_{b}\right) \\
& \left.-k_{b 4}\left(z_{b 2}-z_{b}\right)-c_{d 4}\left(\dot{z}_{d 2}-\dot{z}_{d}\right)-k_{d 4}\left(z_{d 2}-z_{d}\right)\right] .
\end{aligned}
$$

From (9), (10), (11), and (12), the vertical motion equations of the four endpoints of the vehicle body are obtained:

$$
\begin{aligned}
& \dot{z}_{a}=\dot{z}+l_{f} \dot{\theta}+\frac{1}{2} d \dot{\varphi}, \\
& \dot{z}_{b}=\dot{z}+l_{f} \dot{\theta}-\frac{1}{2} d \dot{\varphi}, \\
& \dot{z}_{c}=\dot{z}-l_{r} \dot{\theta}+\frac{1}{2} d \dot{\varphi}, \\
& \dot{z}_{d}=\dot{z}-l_{r} \dot{\theta}-\frac{1}{2} d \dot{\varphi} .
\end{aligned}
$$

In comparing this mathematical model with the mathematical model deduced using Newton's second law, both are the same; as a result, the correctness of the BG model is verified.

\section{Model Simplifications and Comparative Analysis}

Figure 2 shows that there are many parts in the physical model; this larger number of parts will affect the subsequent vibration characteristic analysis. Because some parts have less of an effect on vehicle vibration characteristics, these parts can be identified through simulation and analysis and subsequently removed, thereby simplifying the model.

4.1. Model Simplification. The simplified model approach is as follows: according to the change in power of each part in the system over time, the sum of the energy absorption and release of each part at a given period of time is calculated. The relevant calculation formula of the power for each part is calculated by

$$
W(t)=\int P(t) d t
$$

where $W(t)$ represents the sum of the energy absorption and release of each part in the system at a given period of time and $P(t)$ represents the power changes over time of each part.

The above approach is used to judge the activity of each part in the system. This activity is represented by the sum of energy absorption and release by each part at a given period of time as a percentage of the sum of energy absorption and release by the whole system (all parts) at this period of time. The low activity of some parts illustrates that the impact of the parts on the vehicle vibration characteristics is small; thus, the activity of these less relevant parts is easily identified, and such parts can be removed to simplify the model. 

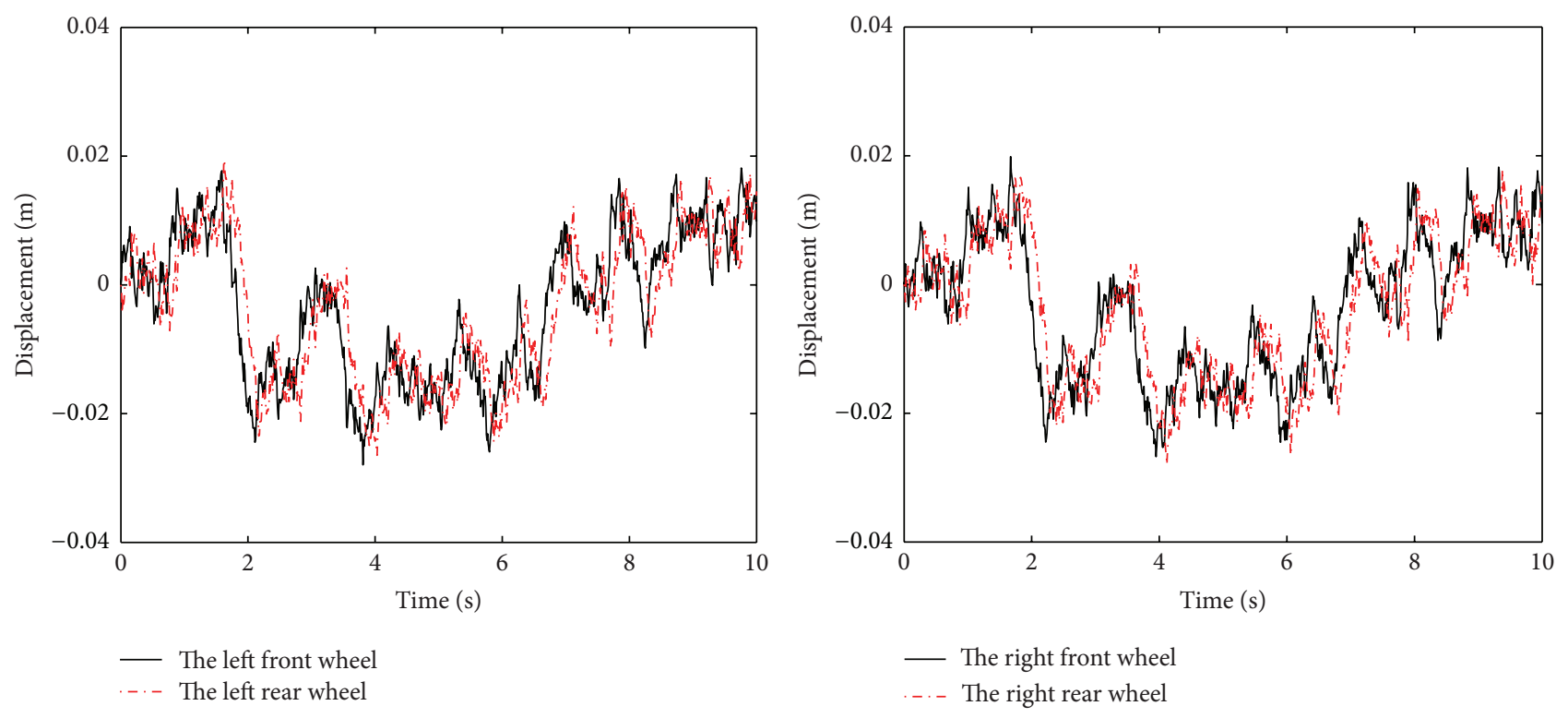

FIGURE 6: Road random input of four wheels.

4.1.1. The Vehicle Parameters. The parameters of the IWMDV model are given as shown in Table 1 .

4.1.2. Time Domain Model of the Road Surface Roughness Excitation. In the vehicle vibration characteristics analysis, the road correlation of the four wheels should be considered. In this paper, a four-wheel correlation time domain model of road roughness excitations is built up [10]. The state equation of the four-wheel related road excitation is given as follows:

$$
\dot{Z}(t)=A Z(t)+B_{0} S(t),
$$

where

$$
\begin{aligned}
& Z(t)=\left[\begin{array}{llllll}
z_{1}(t) & z_{2}(t) & z_{3}(t) & z_{4}(t) & x_{1}(t) & x_{2}(t)
\end{array}\right]^{T} ; \\
& \dot{Z}(t)=\left[\begin{array}{llllll}
\dot{z}_{1}(t) & \dot{z}_{2}(t) & \dot{z}_{3}(t) & \dot{z}_{4}(t) & \dot{x}_{1}(t) & \dot{x}_{2}(t)
\end{array}\right]^{T} ;
\end{aligned}
$$

$A$

$$
\left[\begin{array}{cccccc}
a_{1} & 0 & 0 & 0 & 0 & 0 \\
\frac{e^{-2 \pi n_{00} d} u}{d} & -\frac{u}{d} & 0 & 0 & 0 & 0 \\
-\frac{12 u}{l}+a_{1} & 0 & 0 & 0 & 0 & 0 \\
\frac{e^{-2 \pi n_{00} d} u}{d} & -\left(\frac{12 u}{l}+\frac{u}{d}\right) & 0 & 0 & 0 & 1 \\
-\frac{12 u}{l} & 0 & 0 & 0 & 0 & 1 \\
\frac{72 u^{2}}{l^{2}} & 0 & 0 & 0 & -\frac{12 u^{2}}{l^{2}} & -\frac{6 u}{l}
\end{array}\right]
$$

$B_{0}=\left[\begin{array}{llllll}b_{1} & 0 & b_{1} & 0 & 0 & 0\end{array}\right]^{T}$;

$b_{1}=2 \pi n_{0} \sqrt{S_{q}\left(n_{0}\right) u}$

$a_{1}=-2 \pi n_{00} u$
TABLE 1: Parameters of the IWMDV.

\begin{tabular}{lcc}
\hline Variable & Value & Unit \\
\hline$m_{b}$ & 1580 & $\mathrm{~kg}$ \\
$k_{a 1} / k_{b 1} / k_{c 1} / k_{d 1}$ & $400 / 400 / 400 / 400$ & $\mathrm{kN} / \mathrm{m}$ \\
$k_{a 2} / k_{b 2} / k_{c 2} / k_{d 2}$ & $5120 / 5120 / 5120 / 5120$ & $\mathrm{kN} / \mathrm{m}$ \\
$k_{a 3} / k_{b 3} / k_{c 3} / k_{d 3}$ & $5000 / 5000 / 5000 / 5000$ & $\mathrm{kN} / \mathrm{m}$ \\
$k_{a 4} / k_{b 4} / k_{c 4} / k_{d 4}$ & $80 / 80 / 80 / 80$ & $\mathrm{kN} / \mathrm{m}$ \\
$c_{a 1} / c_{b 1} / c_{c 1} / c_{d 1}$ & $100 / 100 / 100 / 100$ & $\mathrm{~N} \cdot \mathrm{s} / \mathrm{m}$ \\
$c_{a 2} / c_{b 2} / c_{c 2} / c_{d 2}$ & $0 / 0 / 0 / 0$ & $\mathrm{~N} \cdot \mathrm{s} / \mathrm{m}$ \\
$c_{a 3} / c_{b 3} / c_{c 3} / c_{d 3}$ & $0 / 0 / 0 / 0$ & $\mathrm{~N} \cdot \mathrm{s} / \mathrm{m}$ \\
$c_{a 4} / c_{b 4} / c_{c 4} / c_{d 4}$ & $5000 / 5000 / 5000 / 5000$ & $\mathrm{~N} \cdot \mathrm{s} / \mathrm{m}$ \\
$m_{a 1} / m_{b 1} / m_{c 1} / m_{d 1}$ & $80 / 80 / 80 / 80$ & $\mathrm{~kg}$ \\
$m_{a 2} / m_{b 2} / m_{c 2} / m_{d 2}$ & $50 / 50 / 50 / 50$ & $\mathrm{~kg}$ \\
$I_{x} / I_{y}$ & $1100 / 3000$ & $\mathrm{~kg} \cdot \mathrm{m}^{2}$ \\
$d$ & 1.3 & $\mathrm{~m}$ \\
$l_{f} / l_{r}$ & $1.19 / 1.2$ & $\mathrm{~m}$ \\
\hline
\end{tabular}

$S(t)$ is the Gaussian white noise; $S_{q}\left(n_{0}\right)$ is the road roughness coefficient; $n_{0}$ is the reference frequency; $n_{00}$ is the road cutoff spatial frequency; $u$ is the vehicle speed; $l$ is the length of the vehicle; and $l=l_{f}+l_{r}$.

4.1.3. Simulation Analysis. In this paper, the speed $u$ is $50 \mathrm{~km} / \mathrm{h}$, the road excitation simulated is a class B road, the road roughness coefficient is $S_{q}\left(n_{0}\right)=64 \times 10^{-6} \mathrm{~m}^{3}$, the road space cutoff frequency is $n_{00}=0.01 \mathrm{~m}^{-1}$, and the four-wheel correlation input of the road surface roughness excitation is obtained using Matlab/Simulink simulation [11], as shown in Figure 6. 


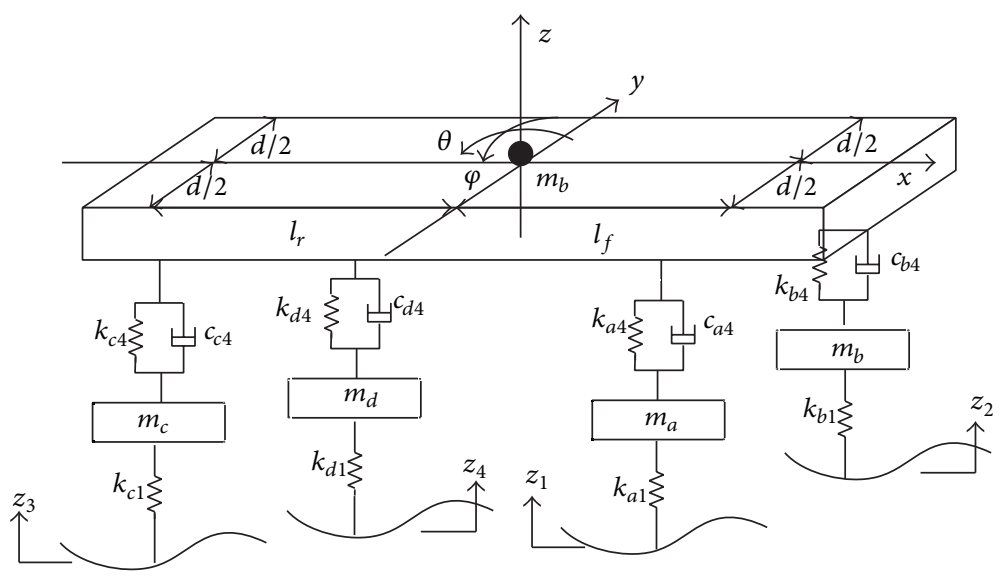

FIGURE 7: The simplified physical model.

TABLE 2: Sum of the energy absorption and release of each part in the system within $10 \mathrm{~s}$.

\begin{tabular}{lcc}
\hline Part & $\begin{array}{c}\text { Sum of the energy } \\
\text { absorption and release } \\
\text { within } 10 \mathrm{~s}\end{array}$ & Percentage $(\%)$ \\
\hline$k_{a 1} / k_{b 1} / k_{c 1} / k_{d 1}$ & $1178.9 / 1351.1 / 1198.2 / 1218.6$ & $6.96 / 7.97 / 7.07 / 7.19$ \\
$k_{a 2} / k_{b 2} / k_{c 2} / k_{d 2}$ & $9.1 / 10.9 / 9.2 / 8.1$ & $0.05 / 0.06 / 0.05 / 0.05$ \\
$k_{a 3} / k_{b 3} / k_{c 3} / k_{d 3}$ & $8.9 / 10.6 / 9 / 7.9$ & $0.05 / 0.06 / 0.05 / 0.05$ \\
$k_{a 4} / k_{b 4} / k_{c 4} / k_{d 4}$ & $180.8 / 334.6 / 185 / 134.8$ & $1.07 / 1.98 / 1.09 / 0.8$ \\
$c_{a 1} / c_{b 1} / c_{c 1} / c_{d 1}$ & $46 / 44.4 / 47.1 / 44.7$ & $0.27 / 0.26 / 0.28 / 0.26$ \\
$c_{a 2} / c_{b 2} / c_{c 2} / c_{d 2}$ & $0 / 0 / 0 / 0$ & $0 / 0 / 0 / 0$ \\
$c_{a 3} / c_{b 3} / c_{c 3} / c_{d 3}$ & $0 / 0 / 0 / 0$ & $0 / 0 / 0 / 0$ \\
$c_{a 4} / c_{b 4} / c_{c 4} / c_{d 4}$ & $578.7 / 611.9 / 543.5 / 469$ & $3.42 / 3.61 / 3.21 / 2.71$ \\
$m_{a 1} / m_{b 1} / m_{c 1} / m_{d 1}$ & $613 / 684.8 / 652.7 / 695.2$ & $3.62 / 4.04 / 3.85 / 4.1$ \\
$m_{a 2} / m_{b 2} / m_{c 2} / m_{d 2}$ & $395 / 436.3 / 420.2 / 444.2$ & $2.33 / 2.58 / 2.48 / 2.62$ \\
$m_{b}$ & 4360.1 & 25.73 \\
\hline
\end{tabular}

Substituting the above road surface roughness excitation into the BG model of the vehicle, the sum of energy absorption and release of each part in the system within $10 \mathrm{~s}$ can be calculated by (39). Then, the power for each part can be calculated as a percentage of the sum of energy absorption and release of the whole system during this period. The statistical data are listed in Table 2.

As observed from the data in Table 2, the percentage of the parts $k_{i 2}, k_{i 3}, c_{i 1}, c_{i 2}$, and $c_{i 3}$ is less than $0.5 \%$, which indicates that the activity of these parts is low. As a result, the impact of these parts on the vehicle vibration characteristics can be ignored, and the model can be simplified and ultimately obtained (as shown in Figure 7). In this simplified model, the mass of $m_{a 2}, m_{b 2}, m_{c 2}$, and $m_{d 2}$ is combined with the mass of $m_{a 1}, m_{b 1}, m_{c 1}$, and $m_{d 1}$, respectively, yielding the combination masses $m_{a}, m_{b}, m_{c}$, and $m_{d}$, respectively, for the whole in-wheel motor as a rigid body.

4.2. Simplified Mathematical Model. The BG model of the simplified physical model is established in this section. Because this model is similar to the BG model shown in Figure 5, we can eliminate the parts with low activity from Figure 5 directly. The simplified BG model can be obtained, which is shown in Figure 8. In this figure, in addition to the symbol $m_{a}, m_{b}, m_{c}$, and $m_{d}$ represent the summation of $m_{a 2} \& m_{a 1}, m_{b 2} \& m_{b 1}, m_{c 2} \& m_{c 1}$, and $m_{d 2} \& m_{d 1}$, respectively; the other symbols are the same as described in Figure 5.

Similarly, according to Figure 8, the state equations of MAS are obtained as follows.

The vertical velocity equation of $m_{a}$ is

$$
\dot{q}_{2}=\dot{q}_{a}-\frac{p_{4}}{m_{a}}
$$

The vertical velocity equation of $m_{b}$ is

$$
\dot{q}_{10}=\dot{q}_{b}-\frac{p_{12}}{m_{b}}
$$

The vertical velocity equation of $m_{c}$ is

$$
\dot{q}_{18}=\dot{q}_{c}-\frac{p_{20}}{m_{c}} .
$$

The vertical velocity equation of $m_{d}$ is

$$
\dot{q}_{50}=\dot{q}_{d}-\frac{p_{52}}{m_{d}}
$$

The vertical force equation of $m_{a}$ is

$$
\dot{p}_{4}=k_{a 1} q_{2}-k_{a 4} q_{8}-c_{a 4} \dot{q}_{8} .
$$




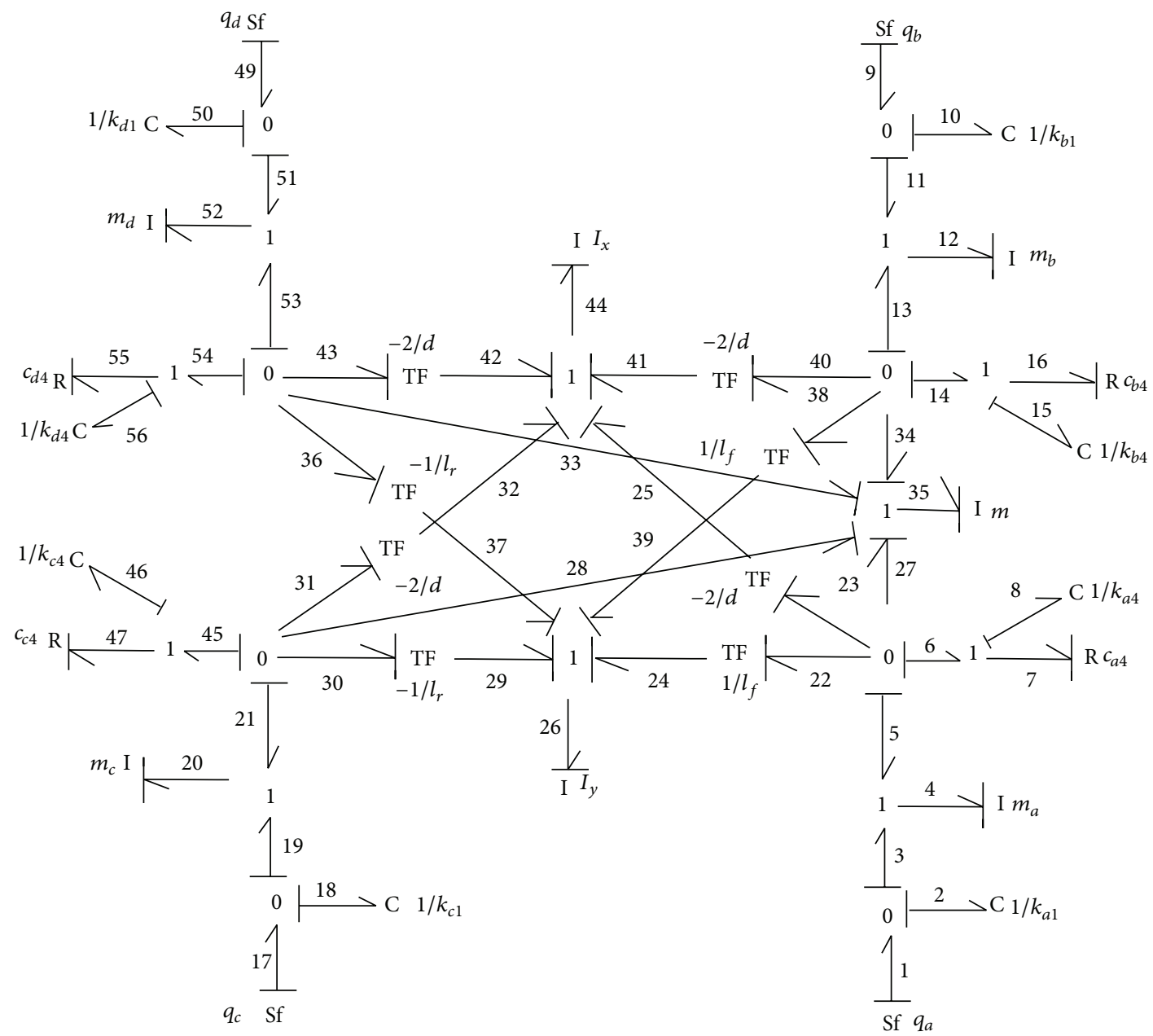

Figure 8: The simplified BG model.

The vertical force equation of $m_{b}$ is

$$
\dot{p}_{12}=k_{b 1} q_{10}-k_{b 4} q_{16}-c_{b 4} \dot{q}_{16} .
$$

The vertical force equation of $m_{c}$ is

$$
\dot{p}_{20}=k_{c 1} q_{18}-k_{c 4} q_{46}-c_{c 4} \dot{q}_{46} .
$$

The vertical force equation of $m_{d}$ is

$$
\dot{p}_{52}=k_{d 1} q_{50}-k_{d 4} q_{55}-c_{d 4} \dot{q}_{55} .
$$

The vertical force equation of the vehicle body is

$$
\begin{aligned}
\dot{p}_{26}= & c_{a 4} \dot{q}_{8}+k_{a 4} q_{8}+c_{b 4} \dot{q}_{16}+k_{b 4} q_{16}+c_{c 4} \dot{q}_{46} \\
& +k_{c 4} q_{46}+c_{d 4} \dot{q}_{55}+k_{d 4} q_{55} .
\end{aligned}
$$

The pitching moment equation of the vehicle body is

$$
\begin{aligned}
\dot{p}_{44} & =\frac{d}{2}\left(c_{a 4} \dot{q}_{8}+k_{a 4} q_{8}+c_{c 4} \dot{q}_{46}+k_{c 4} q_{46}-c_{b 4} \dot{q}_{16}\right. \\
& \left.-k_{b 4} q_{16}-c_{d 4} \dot{q}_{55}-k_{d 4} q_{55}\right) .
\end{aligned}
$$

The roll torque equation of the vehicle body is

$$
\begin{aligned}
\dot{p}_{26}= & l_{f}\left(c_{a 4} \dot{q}_{8}+k_{a 4} q_{8}+c_{b 4} \dot{q}_{16}+k_{b 4} q_{16}\right) \\
& -l_{r}\left(c_{c 4} \dot{q}_{46}+k_{c 4} q_{46}+c_{d 4} \dot{q}_{55}+k_{d 4} q_{55}\right) .
\end{aligned}
$$

The vertical velocity equations of the four endpoints of the vehicle body are the same as (9), (10), (11), and (12).

4.3. Comparison and Analysis. Consistent with the previous model parameters, the contrast results of the four-wheel dynamic tire loads, the four-wheel suspension dynamic travel, the body vertical acceleration (BVA), the body pitching angle acceleration (BPAA), and the body roll angle acceleration (BRAA) between the MBS and the MAS are obtained by using Matlab/Simulink simulation analysis. The simulation results are depicted in Figures 9-13. Figure 9 includes four figures: the contrast results of the left front wheel tire dynamic load (LFWTDL), the right front wheel tire dynamic load (RFWTDL), the left rear wheel tire dynamic load (LRWTDL), and the right rear wheel tire dynamic load (RRWTDL). 

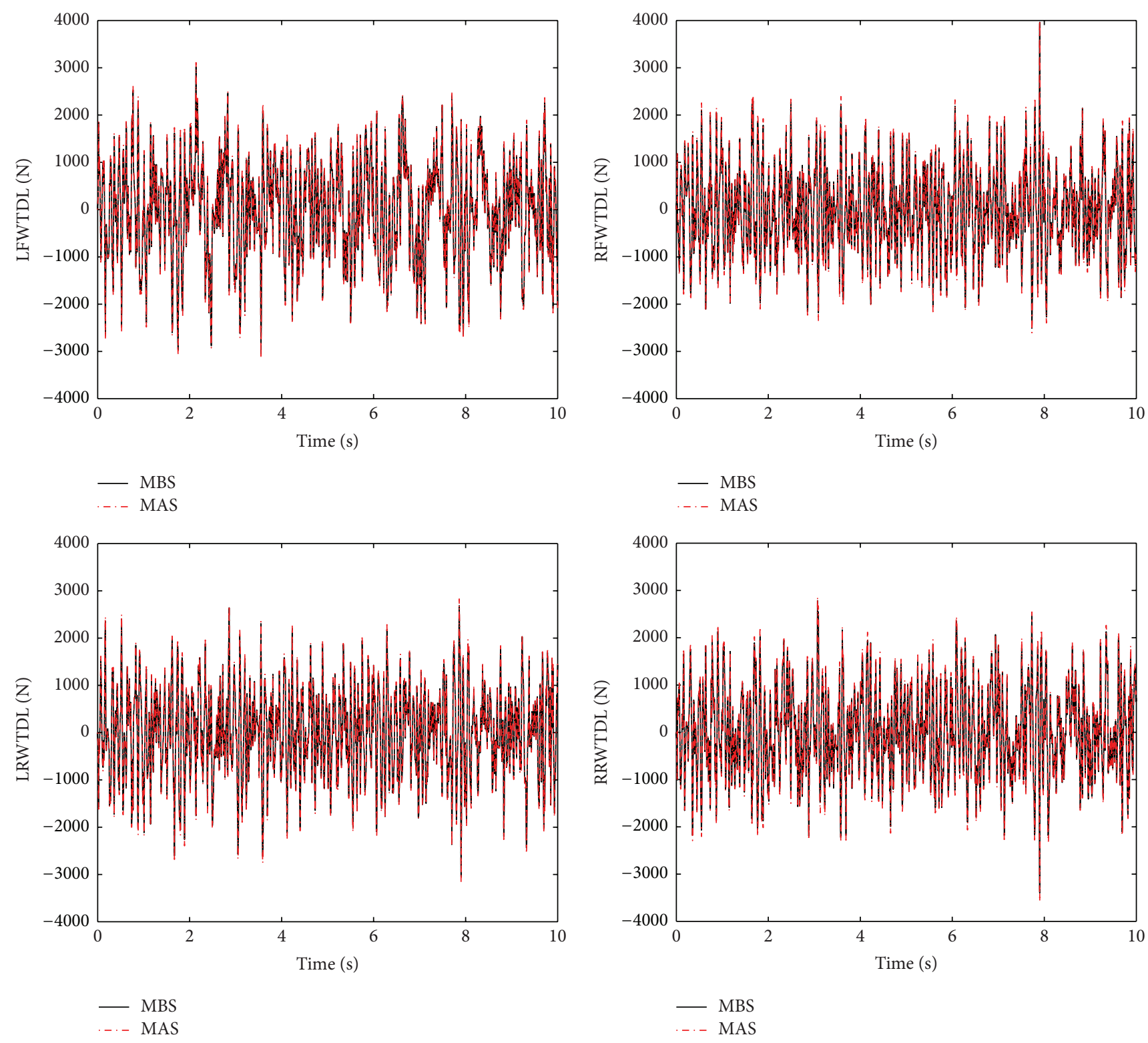

FIGURE 9: Contrast results of the tire dynamic loads.

Figure 10 includes four figures: the contrast results of the left front wheel suspension dynamic course (LFWSDC), the right front wheel suspension dynamic course (RFWSDC), the left rear wheel suspension dynamic course (LRWSDC), and the right rear wheel suspension dynamic course (RRWSDC).

From the above simulation results, the root mean square values (RMSVs) of the dynamic tire loads, suspension dynamic course, BVA, BPAA, and BRAA between MBS and MAS are compared and analyzed; the contrasting data of the MBS and MAS are listed in Table 3.

The statistical data in Table 3 indicates that the change in vibration response variables of the MBS and MAS as well as the RMSV of the LFWTDL, RFWTDL, LRWTDL, and RRWTDL is larger relatively, whereas changes in the other vibration response variables are smaller. The maximal
TABLE 3: Contrasting data of the MBS and MAS.

\begin{tabular}{lccc}
\hline RMSV & MBS & MAS & Difference (\%) \\
\hline RFWTDL (N) & 885.6 & 934.0 & 5.47 \\
LFWTDL (N) & 1025.6 & 1052.6 & 2.63 \\
RRWTDL (N) & 914.2 & 957.6 & 4.75 \\
LRWTDL (N) & 911.9 & 950.5 & 4.23 \\
RFWSDC (m) & 0.0032 & 0.0033 & 3.13 \\
LFWSDC (m) & 0.0058 & 0.0058 & 0 \\
RRWSDC (m) & 0.0036 & 0.0037 & 2.78 \\
LRWSDC (m) & 0.0028 & 0.0028 & 0 \\
BVA (m/s $\left./ \mathrm{s}^{2}\right)$ & 0.3167 & 0.3214 & 1.48 \\
BPAA (rad $\left./ \mathrm{s}^{2}\right)$ & 0.5380 & 0.533 & 0.93 \\
BRAA $\left(\mathrm{rad} / \mathrm{s}^{2}\right)$ & 0.5937 & 0.6099 & 2.73 \\
\hline
\end{tabular}



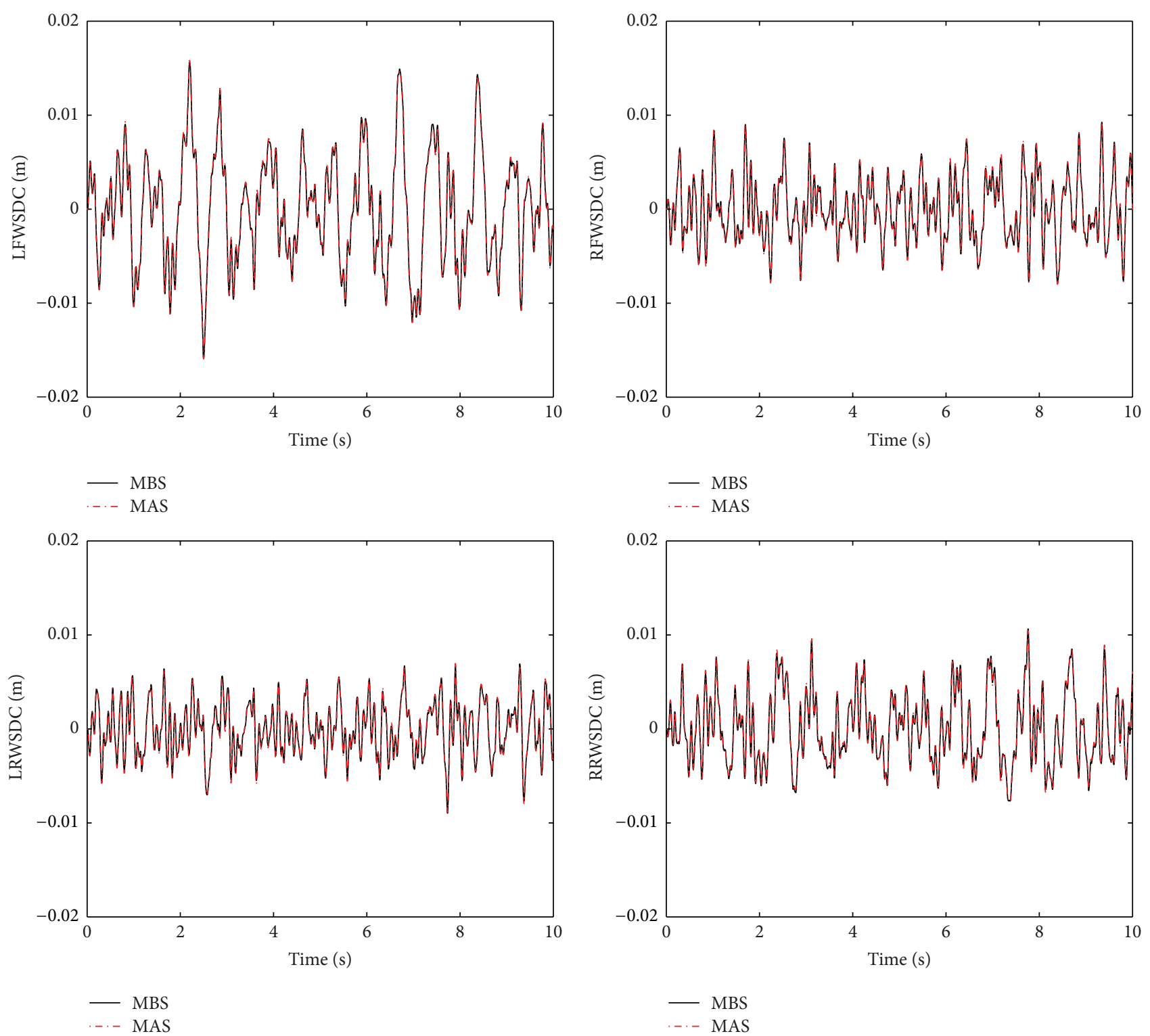

- MBS
-.. MAS

Figure 10: Contrast results of the suspension dynamic course.

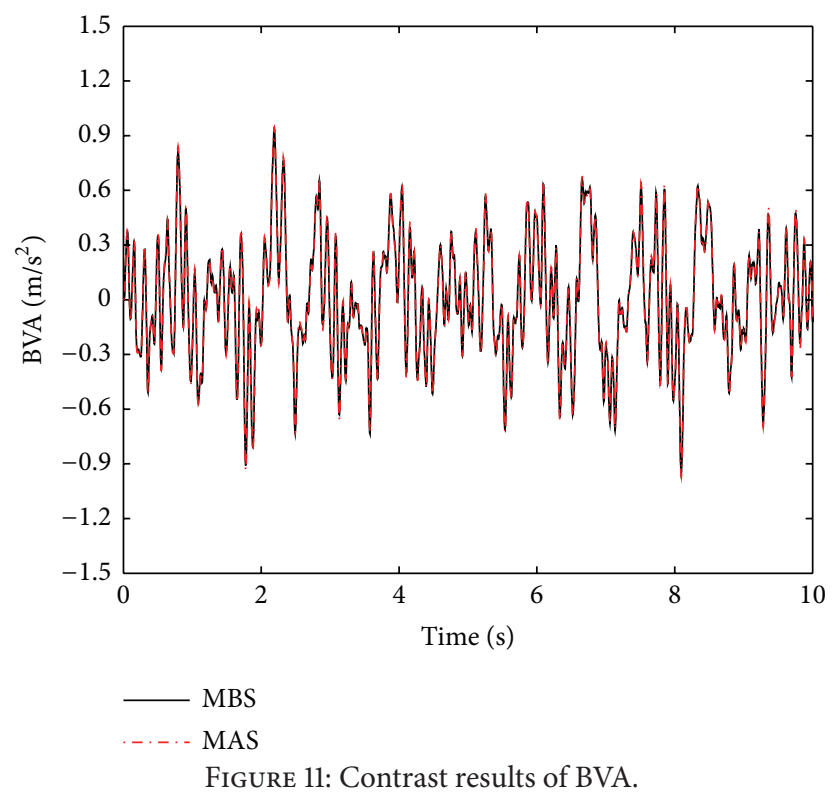




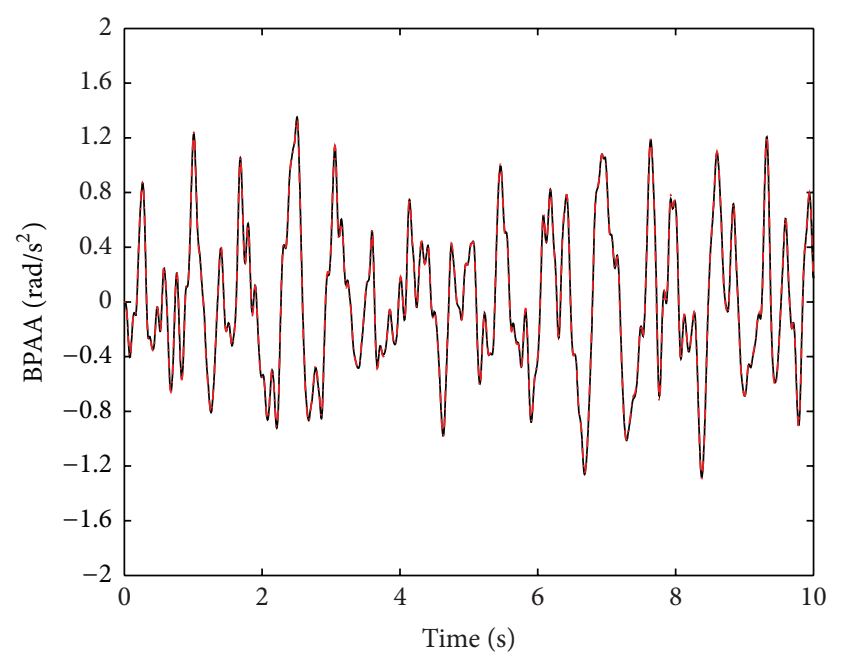

MBS

FIGURE 12: Contrast result of BPAA.

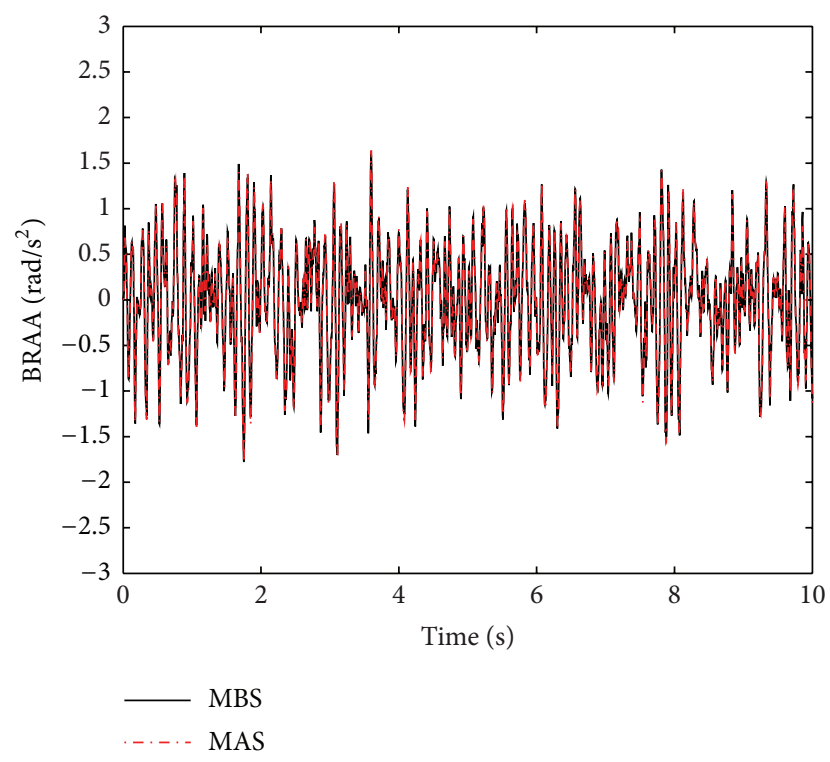

FIGURE 13: Contrast results of BRAA.

difference is only $5.47 \%$. These observations illustrate the reliability of the MAS based on activity.

\section{Conclusions}

Based on the modeling and analysis of the IWMDV vibration characteristics, the following conclusions can be drawn:

(1) The BG theory is found to be clearly superior in performing dynamics analysis. The BG method is a physical object-oriented method, and the BG modular modeling approach simplifies the modeling of the complex system; thus, the BG model is advantageous for performing model modification. Using the BG model, the state equations of the model are deduced directly, which is beneficial for subsequent analysis of the IWMDV vibration characteristics.

(2) By calculating the activity of each part in the system, the parts that are less influential to the IWMDV vibration characteristics can be effectively identified and then removed to simplify the model. The reliability of the MAS is verified based on the contrastive analysis.

(3) Without considering the in-wheel motor excitation, the in-wheel motor can be analyzed as a whole rigid body, and the tires can be simplified as a spring in the vehicle vibration analysis.

In summary, this study can provide a method for the modeling and simulation of the vibration characteristics of the IWMDV.

\section{Conflict of Interests}

The authors declare that there is no conflict of interests regarding the publication of this paper.

\section{Acknowledgments}

This research is sponsored by National Natural Science Foundation of China (Grant no. 51405273) and supported by Shandong Province Higher Educational Science and Technology Program (Grant no. J14LB08) and Doctoral Program for Shandong University of Technology (Grant no. 4041-413039).

\section{References}

[1] D. C. Karnopp, D. L. Margolis, and R. C. Rosenberg, System Dynamics: Modeling and Simulation of Mechatronic Systems, John Wiley \& Sons, New York, NY, USA, 2006.

[2] L. Louca, J. Stein, G. Hulbert et al., "Proper model generation: an energy-based methodology," in Proceedings of the 3rd International Conference on Bond Graph Modeling and Simulation (ICBGM '97), vol. 29, pp. 44-49, Phoenix, Ariz, USA, January 1997.

[3] J. R. Chen, Y. Lin, M. K. Liu, and Y. H. Peng, "Application of bond graph theory in vibration analysis of vehicles," Automotive Engineering, vol. 15, no. 1, pp. 26-33, 1993.

[4] W. Tao, W. Qing, and L. Yon, "Modeling and simulation for powertrain of electric vehicle based on bond graph," Transactions of the Chinese Society of Agricultural Engineering, vol. 27, no. 12, pp. 64-68, 2011.

[5] Q. Zhang, Analysis of ride comfort of all terrain crane based on bond theory [Ph.D. thesis], Taiyuan University of Science and Technology, Taiyuan, China, 2014.

[6] B. Ma, D. Wu, H. Wen et al., "Fuzzy logic control research of hydro-pneumatic suspension system of multi-axle vehicle by power bond graph theory," Journal of Shihezi University (Natural Science), vol. 33, no. 2, pp. 258-264, 2015.

[7] M. Zeraoulia, M. E. H. Benbouzid, and D. Diallo, "Electric motor drive selection issues for HEV propulsion systems: a comparative study," IEEE Transactions on Vehicular Technology, vol. 55, no. 6, pp. 1756-1764, 2006.

[8] R. Loureiro, R. Merzouki, and B. O. Bouamama, "Bond graph model based on structural diagnosability and recoverability analysis: application to intelligent autonomous vehicles," IEEE Transactions on Vehicular Technology, vol. 61, no. 3, pp. 986-997, 2012. 
[9] Z. Wang, Y. Gao, and Y. Wang, "The transformation of system state-space equations based on bond graph theory," Mechanical Science and Technology, vol. 18, no. 1, pp. 54-56, 1999.

[10] Z. Lijun and Z. Tianxia, "General nonstationary random input model of road surface with four wheels correlated," Journal of Vibration and Shock, vol. 18, no. 1, pp. 54-56, 1999.

[11] S. Chen, J. Li, and Z. Fang, "Modeling and simulation of Automotive suspension system based on bond graph," Computer Simulation, vol. 24, no. 9, pp. 245-249, 2007. 


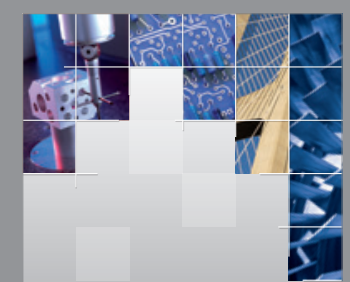

\section{Enfincering}
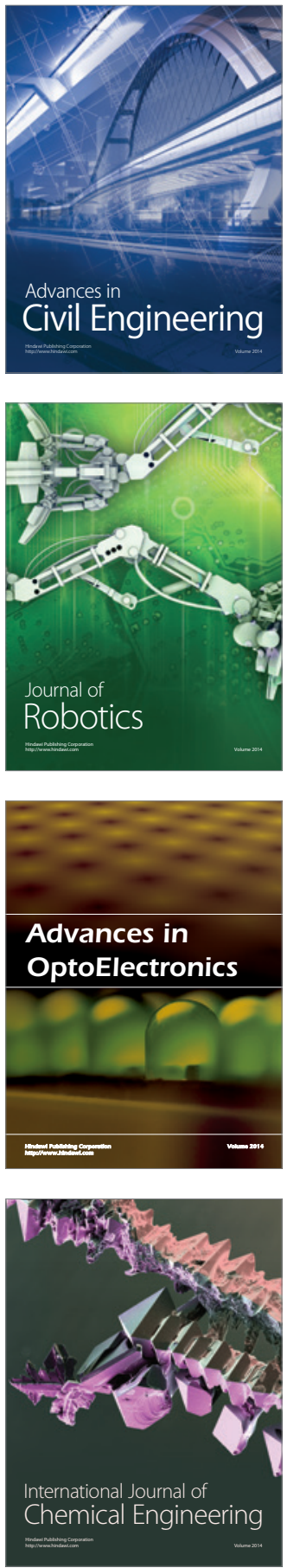

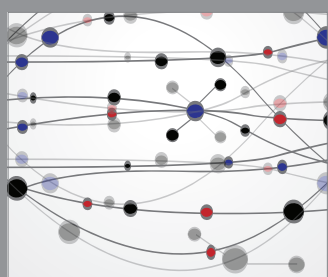

The Scientific World Journal

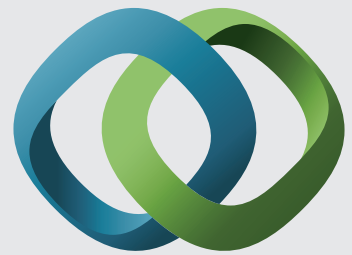

\section{Hindawi}

Submit your manuscripts at

http://www.hindawi.com
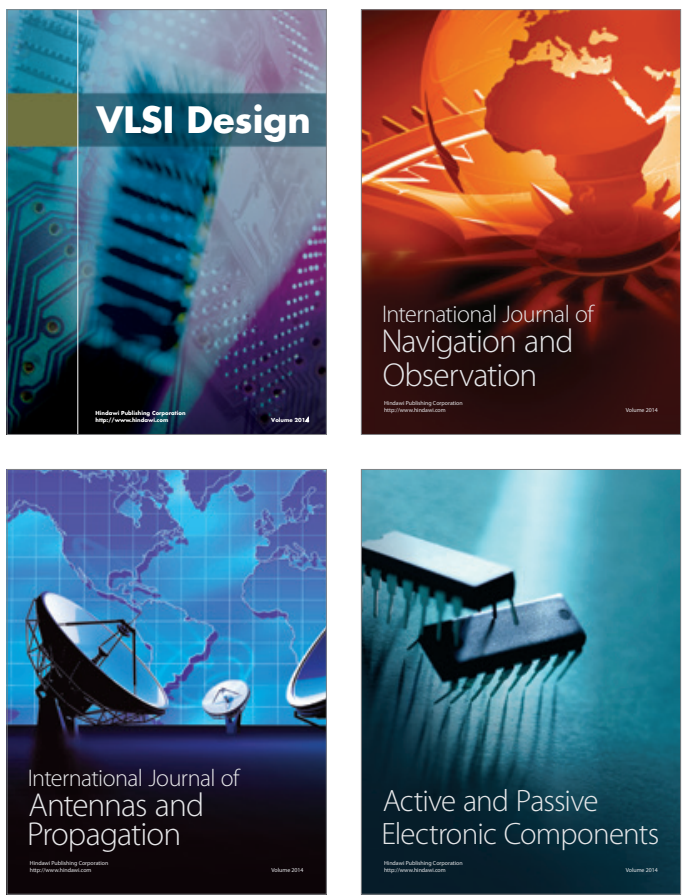
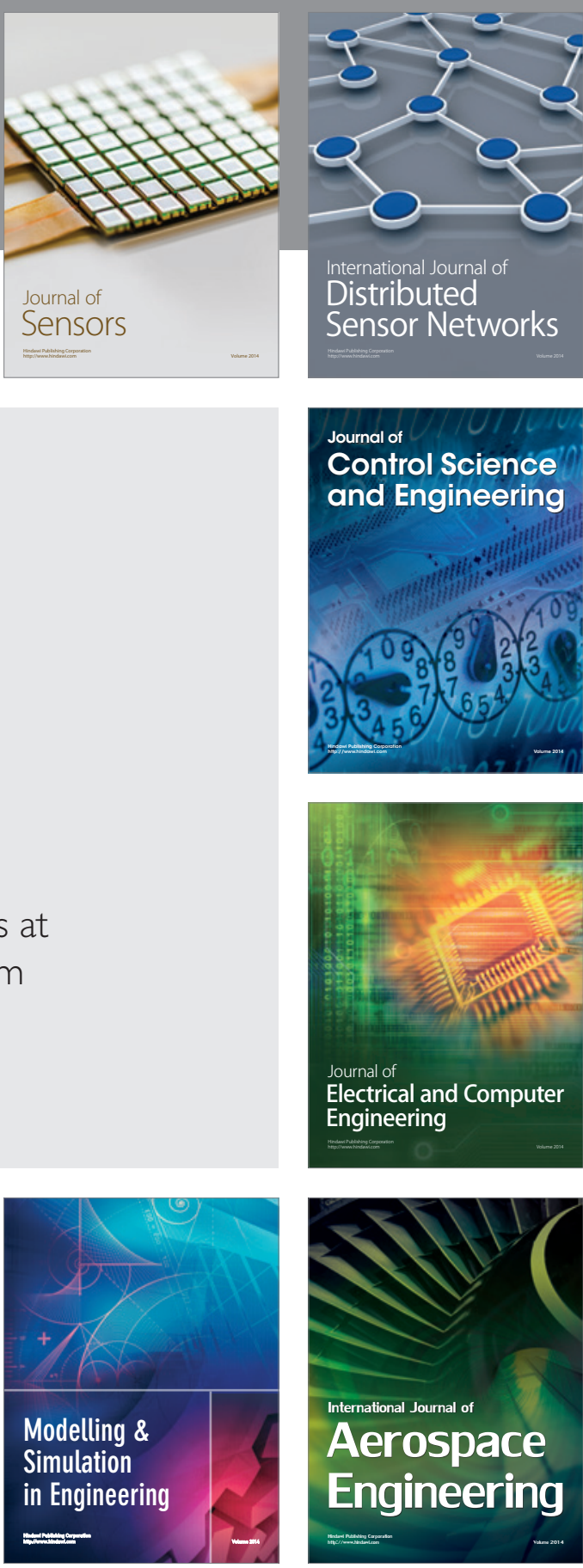

International Journal of

Distributed

Sensor Networks

Journal of

Control Science

and Engineering
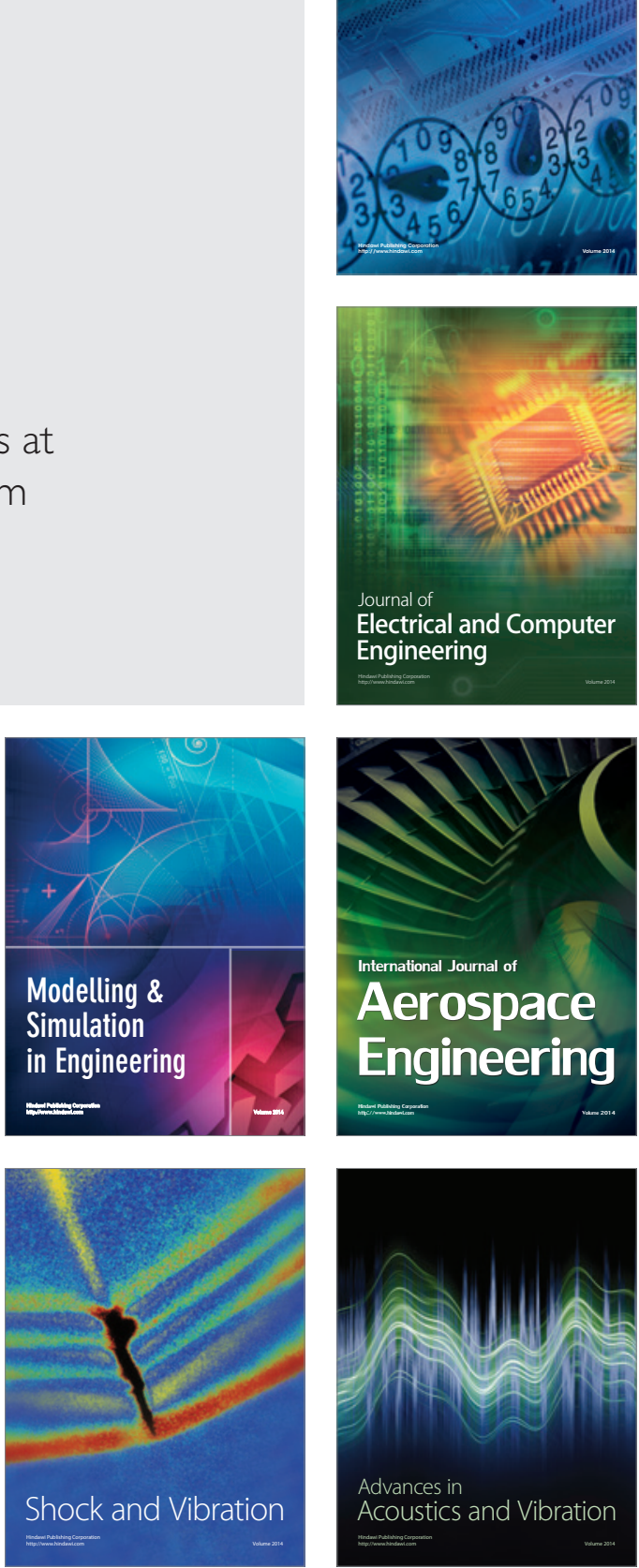